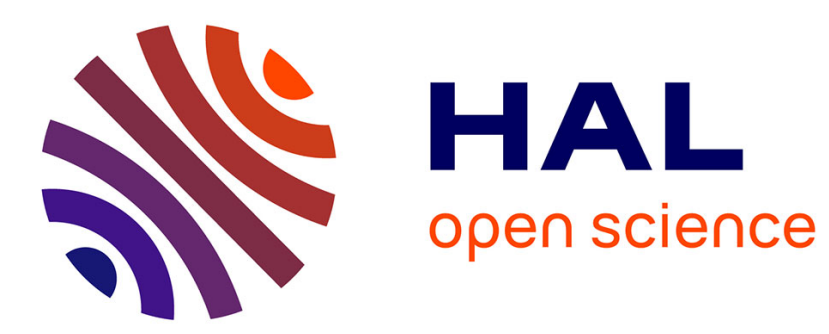

\title{
La constitution de classes de niveau dans les collèges: les effets pervers d'une pratique à visée égalisatrice
}

\author{
Marie Duru-Bellat, Alain Mingat
}

\section{To cite this version:}

Marie Duru-Bellat, Alain Mingat. La constitution de classes de niveau dans les collèges: les effets pervers d'une pratique à visée égalisatrice. Revue française de sociologie, 1997, 38 (4), pp.759-789. hal-03207869

\section{HAL Id: hal-03207869 \\ https://hal.science/hal-03207869}

Submitted on 27 May 2021

HAL is a multi-disciplinary open access archive for the deposit and dissemination of scientific research documents, whether they are published or not. The documents may come from teaching and research institutions in France or abroad, or from public or private research centers.
L'archive ouverte pluridisciplinaire HAL, est destinée au dépôt et à la diffusion de documents scientifiques de niveau recherche, publiés ou non, émanant des établissements d'enseignement et de recherche français ou étrangers, des laboratoires publics ou privés. 
La constitution de classes de niveau dans les collèges ; les effets pervers d'une pratique à visée égalisatrice

Marie Duru-Bellat, Monsieur Alain Mingat

\section{Citer ce document / Cite this document :}

Duru-Bellat Marie, Mingat Alain. La constitution de classes de niveau dans les collèges ; les effets pervers d'une pratique à visée égalisatrice. In: Revue française de sociologie, 1997, 38-4. Le suicide un siècle après Durkheim. pp. 759-789; doi : $10.2307 / 3322627$

https://www.persee.fr/doc/rfsoc_0035-2969_1997_num_38_4_4667

Fichier pdf généré le 23/04/2018 


\title{
Resumen
}

Marie Duru-Bellat, Alain Mingat : La constitución de clases de nivel en los colegios : los efectos perversos de una práctica con miras igualatorias.

En base a datos recogidos entre 1989 y 1992 por la DEP (Sección del estimación y del prospectiva del ministerio de la Instrucción Publica) a nivel de colegio, este texto se propone evaluar la frecuencia y los efectos de la práctica en la elaboración de clases de un nivel determinado. Luego, dirigiéndose primero a operacionalizar este concepto, para después producir una estimación, en la cual se expliscita el caracter arbitrario del porcentajede colegios que constituyen este tipo de clase a la entrada en sexto año. También, interesa al papel desempeñado por el reagrupamiento de los alumnos en función del idioma estudiado, en la composición de clases de nivel en el seno de los establecimientos. Por último un análisis modelizado de los efectos del modo del agrupamiento sobre las progresiones escolares de los alumnos en el transcurso de los dos primeros años del colegio es propuesto, así como una evaluación de ese factor contextual sobre la orientación al finalizar el quinto año. Se concluye de estos análisis que las clases de nivel (o los otros dispositivos con la misma finalidad tales como los ciclos de tres años), en esta etapa de la escolaridad, tiene mas bien la tendencia a acentuar las desigualdades entre alumnos.

\section{Résumé}

Ce texte propose, sur la base de données recueillies par la DEP au niveau du collège entre 1989 et 1992, d'évaluer la fréquence et les effets de la pratique de constitution de classes de niveau. II s'attache d'abord à opérationnaliser ce concept, puis produit une estimation, dont on explicite le caractère arbitraire, du pourcentage de collèges qui constituent ce type de classe à l'entrée en sixième. II s'intéresse au rôle joué par le regroupement des élèves en fonction de la langue étudiée, dans la constitution de classes de niveau au sein des établissements. Enfin, une analyse modélisée des effets du mode de groupement sur les progressions scolaires des élèves au cours des deux premières années du collège est proposée, ainsi qu'une évaluation de ce facteur contextuel sur l'orientation en fin de cinquième. II ressort de ces analyses que les classes de niveau (ou les autres dispositifs de même finalité tels que les cycles en trois ans) ont plutôt, à ce stade de la scolarité, tendance à accentuer les inégalités entre élèves.

\begin{abstract}
Marie Duru-Bellat, Alain Mingat : Multi-level classrooms in French secondary schools : the perverse effects of a supposedly equalitarian practice.

This text aims to evaluate the frequency and the effects of multi-level classrooms. It is based on data collected by the DEP (ministry of Education department working on future evaluation) in French secondary schools between 1989 and 1992. The first objective is to put this concept into operation and then generate an estimation (stating its arbitrary characteristic) of the percentage of secondary schools which form this type of classroom in the first year (age 11). The article then looks into the role played by grouping pupils according to the language studied to form the multi-level classes. Finally, a modelled analysis of the effects of the type of grouping on the academic progress of pupils during the first two years at secondary school is given, along with an evaluation of this contextual factor on schooling advice at the end of the second year. This analysis shows that multi-level classes (or similar systems, such as the three-years cycle in France), in fact, at this stage in education, tend to accentuate inequality between pupils.
\end{abstract}

\section{Zusammenfassung}

Marie Duru-Bellat, Alain Mingat : Die Bildung der Niveauklassen in den Mittelschulen : die unerwünschten Nebenwirkungen einer Gleichstellungspraxis.

Dieser Aufsatz möchte auf der Grundlage von Daten der DEP (Prospektivs- und Bewertungsabteilung des Erziehungsministeriums) in den Collèges (Mittelschulen) zwischen 1989 und 1992 die Häufigkeit 
und die Praxiswirkung der Bildung von Niveauklassen bewerten. Er sucht zunächst, das Konzept operativ zu gestalten und anschliessend, eine Schätzung abzuleiten, deren willkürlicher Charakter erklärt wird, zum Prozentsatz der Mittelschulen, die diese Art Klassen bei Beginn der sechsten Schulklasse einführen. Der Aufsatz befasst sich mit der von der Schülerzusammenführung aufgrund der Lernsprache gespielten Rolle in der Bildung von Niveauklassen innerhalb dieser Schulen. Zum Schluss wird eine Modellisierte Analyse der Auswirkungen der Zusammenführungsart gebracht auf die Lernfortschritte der Schüler im Verlauf der beiden ersten Mittelschuljahre und darüber hinaus eine Bewertung dieses kontextuellen Faktors auf die Orientierung am Ende der fünften Schulklasse. Aus diesen Untersuchungen geht hervor, dass die Niveauklassen (oder andere Einrichtungen mit gleichem Zweck wie die Dreijahreszyklen) bei diesem Schulabschnitt eher dazu führen, die Ungleichheit zwischen den Schülern zu fördern. 


\title{
La constitution de classes de niveau dans les collèges ; les effets pervers d'une pratique à visée égalisatrice*
}

\begin{abstract}
RÉSUMMÉ
Ce texte propose, sur la base de données recueillies par la DEP au niveau du collège entre 1989 et 1992, d'évaluer la fréquence et les effets de la pratique de constitution de classes de niveau. Il s'attache d'abord à opérationnaliser ce concept, puis produit une estimation, dont on explicite le caractère arbitraire, du pourcentage de collèges qui constituent ce type de classe à l'entrée en sixième. Il s'intéresse au rôle joué par le regroupement des élèves en fonction de la langue étudiée, dans la constitution de classes de niveau au sein des établissements. Enfin, une analyse modélisée des effets du mode de groupement sur les progressions scolaires des élèves au cours des deux premières années du collège est proposée, ainsi qu'une évaluation de ce facteur contextuel sur l'orientation en fin de cinquième. Il ressort de ces analyses que les classes de niveau (ou les autres dispositifs de même finalité tels que les cycles en trois ans) ont plutôt, à ce stade de la scolarité, tendance à accentuer les inégalités entre élèves.
\end{abstract}

Dans l'analyse de la contribution de l'institution scolaire à la reproduction de la stratification sociale, les sociologues ont, jusqu'au début des années 80 , principalement mis l'accent sur les structures du système et leur correspondance avec les hiérarchies sociales (on pense par exemple à la théorie des deux réseaux de Baudelot et Establet). Le système est apparu de fait diversifié tant dans les contenus dispensés dans chacun de ses segments (filières des collèges, cycles court et long, général et technique, etc.) que dans les caractéristiques des publics qu'il accueille, anticipant ainsi des «destins sociaux» différents. Dans cette perspective, la question de la diversité des structures est censée correspondre à la diversité des élèves, le fait que ceux-ci puissent être néanmoins différents au sein des diverses filières restant perçu comme d'importance seconde.

* Nous tenons à remercier Aletta Grisay (Université de Liège) pour ses remarques très attentives sur une version antérieure de ce texte, ainsi que les rapporteurs de la Revue française de sociologie. Le rapport de recherche dont est tiré ce texte est disponible à l'IREDU (voir Duru-Bellat, Mingat, 1997). 


\section{Revue française de sociologie}

Avec la montée de la scolarisation, puis l'unification progressive des structures au sein de l'enseignement secondaire, le maintien de fortes inégalités sociales de carrières amène les sociologues à s'intéresser aux différences entre élèves : Bourdieu et Passeron soulignent en 1970 que «c'est parce qu'elle est indifférente aux différences» que l'école «donne en fait sa sanction aux inégalités initiales devant la culture ». Cela dit, le caractère implacable de cette fonction de reproduction a d'une certaine façon dissuadé les chercheurs de prendre en compte les contextes de scolarisation ou les comportements des acteurs. Depuis le début des années 80 , et comme l'a montré Cousin (1993), la sociologie a progressivement découvert que le système ne se réduisait pas aux «contraintes qui pèsent sur lui » et que le contexte scolaire n'avait pas un caractère accessoire; dans cette mouvance, la problématique de l'effet-établissement a émergé. On est ainsi amené à s'interroger, comme le faisait Cherkaoui dès 1979 , sur l'influence de l'organisation scolaire sur le déroulement des carrières individuelles et la genèse des inégalités sociales. Ces évolutions se sont manifestées par le développement de nombreuses recherches sur le «local»; elles ont aussi trouvé un double écho tant chez les politiques que chez les acteurs de l'éducation.

Dans ce contexte général, l'idée qu'il convient de traiter différemment les élèves, si l'on entend leur faire atteindre des objectifs communs, est devenue centrale dans les conceptions «pédagogiquement correctes» du moment. Les acteurs se sont emparés de la justification de la notion de pédagogie différenciée, sous-jacente au thème de l' "indifférence aux différences» et ont développé la notion de «discrimination positive». Mais la question des effets de ces traitements pédagogiques différenciés, sur les acquisitions ou les attitudes des élèves, est restée, en France, très peu documentée.

À cet égard, il est apparu intéressant d'exploiter les données collectées par la Direction de l'évaluation et de la prospective du ministère de l'Éducation (DEP), entre 1989 et 1992, visant initialement à évaluer les effets de ce que l'on a appelé le «cycle aménagé», à savoir l'organisation sur 3 ans des deux premières classes du collège. De nombreuses informations concernant un éventail large d'acquis chez les élèves et leur orientation à l'issue du cycle ont été collectées, ainsi que des observations sur les «traitements pédagogiques » auxquels ces élèves ont été exposés; ceci sur 212 collèges et plus de 20000 élèves. Cet échantillon n'est pas à proprement parler représentatif de l'ensemble des collèges français; il compte un peu plus de collèges situés en Zones d'éducation prioritaire $(20 \%$ contre $14 \%$ ) et moins de collèges ruraux $(11,3 \%$ contre $21,6 \%)$. Au niveau individuel, les collèges de l'échantillon comptent un peu plus d'enfants d'ouvriers $(+5 \%)$ et d'élèves en retard $(+8 \%)$ que la moyenne nationale. Dans la mesure où l'on s'intéresse surtout à identifier : 1) des variétés de pratiques d'établissements; et 2) les conséquences associées à des variétés de contexte sur les acquisitions et les carrières des élèves, on peut considérer que cette particularité de l'échantillon n'est pas rédhibitoire. 
Les questions principales auxquelles nous avons tenté de répondre en réexploitant ces données sont les suivantes : 1) dans quelle mesure (avec quelle intensité et par quels moyens, implicites ou explicites) les collèges répondent-ils aux caractéristiques variées de leurs élèves en constituant de fait des classes de niveau; et 2) dans quelle mesure les progressions et les orientations des élèves sont-elles affectées par les caractéristiques de la classe fréquentée et en particulier son niveau moyen et son degré d'hétérogénéité. Ces questions sont en France relativement tabou; et même si le sens commun des parents d'élèves les porte à soupçonner l'existence de classes de niveau, par le truchement des langues ou des options notamment, les données factuelles sur cette question sont très rares. Cela n'empêche pas les pédagogues d'avoir sur cette question une opinion assez tranchée, à savoir le caractère nocif de l'hétérogénéité des classes, en particulier pour les élèves faibles. Les résultats présentés ici montrent d'une part que les collèges ont bien tendance à grouper leurs élèves en fonction de leur niveau initial et d'autre part que ceci a des effets négatifs tant en termes de progression moyenne que sous l'angle des inégalités entre élèves. Ces résultats sont globalement convergents avec une abondante littérature anglo-saxonne qui laisse à penser que les acquis et les attitudes des élèves sont affectés par la façon dont ils sont regroupés dans les classes.

\section{Une question non anodine}

La plupart des recherches, majoritairement anglo-saxonnes, sur les modes de groupement d'élèves concluent que la constitution de groupes de niveau a peu, ou pas, d'impact sur le niveau moyen de l'ensemble des élèves, quels que soient le niveau d'enseignement ou la discipline enseignée (voir notamment Good, Marshall, 1984 ; C. Kulik, J. Kulik, 1982 ; Pallas et al., 1994; Slavin, 1987a et b). En d'autres termes, la présence d'élèves de niveaux différents dans un cours n'entraînerait donc pas d'effet moyen consistant (positif ou négatif) sur les acquisitions des élèves.

Cela dit, au-delà de ce faible effet moyen, la stratification par niveau aurait des effets diversifiés selon le niveau initial des élèves : l'analyse des progressions montre qu'elles sont plus marquées dans les groupes forts, et plus ténues dans les groupes faibles. Cet effet d'interaction amène donc à conclure que le groupement par niveau est significativement nuisible aux progressions des élèves faibles et favorable à celles des élèves forts (par référence à un contexte de classe hétérogène).

Cette tendance générale reste néanmoins discutée, notamment aux ÉtatsUnis, où l'on questionne même la possibilité technique d'isoler un effet spécifique du mode de groupement, en tenant correctement sous contrôle statistique le niveau initial des élèves des différents groupes (Slavin, 1987a et $b, 1990)$. En effet, les élèves placés dans les groupes forts diffèrent 


\section{Revue française de sociologie}

vraisemblablement de leurs condisciples placés dans les groupes faibles par d'autres caractéristiques personnelles qui contribueraient à expliquer leur meilleure progression. Les Britanniques sont plus affirmatifs quant à l'effet divergent des groupes de niveau; des estimations très fines ont été réalisées (voir par exemple Kerckhoff, 1986), prenant en compte également le type d'école fréquenté (de type grammar, secondary-modern ou comprehensive), ce qui est heuristique pour isoler l'effet spécifique du groupement : en effet, globalement, les élèves progressent plus dans ces écoles d' «élite» que sont les grammar, et le niveau moyen des groupes faibles de ces écoles est parfois supérieur à celui des groupes forts des secondarymodern ou des comprehensive; or, quel que soit le niveau moyen « réel» des groupes considérés dans ces contextes différents comme forts ou au contraire faibles, les progressions s'y avèrent bien, en moyenne, respectivement plus fortes ou plus faibles; ceci suggère l'existence d'effets d'étiquetage.

En France, où la constitution de groupes ou de classes de niveau est beaucoup moins formalisée que dans les pays anglo-saxons (si ce n'est dans le second cycle du second degré par l'intermédiaire des séries), l'appréhension de cette pratique va s'avérer du même coup plus complexe, puisque l'on ne peut se fonder sur les déclarations des acteurs. Il faudra donc chiffrer (non sans difficulté, nous y reviendrons) l'hétérogénéité existant de fait dans les classes, le mode de groupement étant alors considéré comme une variable continue. Les rares recherches existantes concluent en général à l'absence d'effet négatif d'une certaine hétérogénéité. Ainsi, au niveau du cours préparatoire, l'hétérogénéité de la classe (mesurée par la dispersion au test initial) aurait un effet moyen positif et significatif (Mingat, 1984, 1987). Plus précisément, l'hétérogénéité profiterait principalement aux élèves inférieurs à la moyenne de la classe; pour les élèves légèrement supérieurs au niveau moyen, il y aurait compensation entre l'effet positif de l'hétérogénéité et l'effet négatif associé à une position audessus de la moyenne de la classe, alors que pour les élèves les meilleurs, il n'y aurait plus compensation : même si l'hétérogénéité les favorise, ils seraient plus fortement défavorisés de par leur position supérieure au niveau moyen de la classe dans laquelle ils se trouvent. Néanmoins, pour un élève donné, il serait au total préférable d'être scolarisé dans une classe dont le niveau moyen est élevé (mais si possible hétérogène), car plus le niveau de la classe est élevé, plus l'élève est dans une position relative faible et plus ses acquisitions sont, toutes choses égales par ailleurs, meilleures.

Au niveau du collège, l'impact moyen de l'hétérogénéité de la classe serait limité mais, selon les travaux, apparaît plutôt positif (PelnardConsidère, 1985) ou plutôt négatif (Duru-Bellat, Mingat, 1988). Plus récemment, on a observé, dans certains collèges, une relation positive entre la progression des élèves et la constitution de classes homogènes (Grisay, 1990); pourtant, le constat inverse est fait sur des données plus nombreuses (échantillon de 100 collèges), sur lesquelles l'hétérogénéité de la popula- 
tion de l'établissement (en termes de milieu social ou de nationalité) est associée à des meilleurs progressions chez les élèves (Grisay, 1993).

Plus constante et plus nette est l'observation, à nouveau, d'effets divergents : un environnement homogène est favorable aux élèves dont le niveau initial est bon, alors que les élèves moyens ou faibles tirent un meilleur bénéfice d'une scolarisation en milieu hétérogène (Grisay, 1990). Une certaine hétérogénéité est donc associée, dans la plupart des travaux, à une réduction des écarts entre les forts et les faibles. Réciproquement, un environnement homogène et l'organisation de classes de niveau (pratique appréhendée par les déclarations des acteurs) seraient associés à un accroissement significatif des clivages de rendement entre élèves pendant les deux premières années du collège (Grisay, 1993). L'hétérogénéité du contexte d'enseignement jouerait donc en interaction avec le niveau initial des élèves, l'appartenance à un groupe de niveau ayant un léger effet positif sur les acquis des bons élèves et un léger effet négatif sur les plus faibles; la répartition par niveau aurait donc tendance à accroître le rendement des groupes «forts» et à diminuer celui des groupes «faibles».

Pour tenter de comprendre les processus sous-jacents à ces effets que l'on peut juger pervers (puisqu'ils vont à l'inverse des objectifs affichés) des groupes de niveau, deux pistes principales ont été explorées.

\section{Une «instruction» différente en quantité et en qualité}

Le principe de base des groupes de niveau est qu'ils sont censés accueillir des élèves différents auxquels il s'agit d'adapter les contenus et la pédagogie. De fait, de nombreuses recherches anglo-saxonnes montrent que les enseignants modulent les contenus et les pratiques pédagogiques en fonction du niveau supposé des élèves ; par exemple, à programme identique, les élèves des «bonnes filières » reçoivent un enseignement abstrait, centré sur la discipline, alors que ceux jugés «faibles » se voient délivrer un enseignement concret, centré sur la relation maître-élèves (Keddie, 1971). Des observations faites en classe montrent par exemple qu'une question identique, posée par des élèves appartenant à un groupe faible ou à un groupe fort, est l'objet d'un traitement différent de la part des enseignants : à une demande d'explication, on répond aux élèves des groupes forts par une démonstration abstraite, alors qu'aux élèves des groupes faibles on donne un exemple de plus. Certes, ce faisant, les enseignants ne font que se conformer à la philosophie implicite de ce mode de groupement, à savoir que les élèves sont dotés d'aptitudes inégales nécessitant des traitements pédagogiques spécifiques, mais inconsciemment, ils offrent aux meilleurs élèves les plus grandes chances de s'améliorer encore, en multipliant les interactions, les renforcements, les stimulations.

Dans certains cas, on assisterait à une véritable «dérive du curriculum » (Grisay, 1993b) : dans la classe, l'adhésion de l'enseignant à une pédagogie 


\section{Revue française de sociologie}

différenciée se traduirait par une diversification non pas seulement des moyens offerts à l'élève pour atteindre les objectifs, mais aussi des objectifs eux-mêmes, des défis plus modestes étant proposés aux élèves les plus faibles. Ceci se traduit, dans le quotidien de la classe, par une utilisation du temps différente : les différentes recherches américaines, qui ont analysé la qualité de l'instruction dispensée aux élèves de groupes forts et faibles, concluent généralement que les groupes de niveau faible reçoivent une instruction significativement inférieure en qualité à celle dispensée aux élèves des groupes forts (Gamoran, Mare, 1989; Oakes, 1982). Les professeurs couvrent également moins le programme dans les classes plus faibles. Au total, les effets des groupes de niveau sur les acquisitions des élèves s'expliqueraient donc par le fait qu'ils amènent les maîtres à moduler la quantité, le rythme ou encore la qualité des activités d'instruction.

\section{Des attentes «autoréalisatrices»}

La seconde famille de processus susceptibles d'expliquer les effets des groupes de niveau est de type psychosocial. On souligne que les groupes de niveau ou les différentes sections (Cherkaoui, 1979) constituent des contextes sociaux au sein desquels les élèves évaluent leurs propres performances et intériorisent les normes scolaires, et apprennent à nourrir telle ou telle ambition concernant leurs performances à venir.

On sait en effet que les appartenances catégorielles interviennent pour une large part dans la définition de soi qu'élabore chaque individu. L'assignation à un groupe de niveau participe donc au processus de construction de l'identité sociale de l'enfant. Ceci vaut même si l'élève a été affecté «par erreur» à tel ou tel groupe; les travaux expérimentaux permettent d'affirmer que fournir à un individu des informations le situant dans une catégorie, bon élève, mauvais élève, par exemple, c'est en quelque sorte lui assigner une place ou une position à laquelle, souvent, en dépit de ses expériences ultérieures, il continuera longtemps à se référer.

De fait, la répartition par niveau affecte fortement les attitudes et comportements des élèves eux-mêmes, dans le sens d'un renforcement des différences initiales. Par exemple, Eder et Felmee (1983) ont montré que les différences de conduite des élèves affectés dans des groupes de niveau différents apparaissent progressivement et seront d'autant plus marquées que l'appartenance à ce groupe est durable. Cette distinction prend la forme, dans les groupes faibles, d'une moindre attention (décrochages plus fréquents, plus remarqués par les pairs,... et mieux tolérés par les enseignants). Cette attention moins soutenue que l'on observe dans les groupes faibles est notée quels que soient le niveau individuel de l'enfant et son degré d'attention avant l'assignation au groupe. Dans ce contexte, de véritables normes de groupes s'instaurent, et au total, les groupes de niveau produiraient une polarisation des attitudes favorables ou au contraire 
défavorables à l'école, non sans incidences multiples sur les projets d'études ultérieures, sur l'absentéisme scolaire, sur la discipline et l'engagement dans le travail (Berends, 1995).

En relation dynamique avec ces images d'eux-mêmes que développent les élèves, les éducateurs (parents et enseignants) élaborent également des représentations et des attentes qui s'ancrent dans l'information apportée par l'assignation à tel ou tel groupe. Le placement dans un groupe symbolise en effet des représentations partagées sur les qualités et les compétences des membres du groupe, indépendamment de leurs compétences effectives. Dans cette perspective, les élèves des meilleurs groupes n'apprendraient pas forcément plus, mais seraient traités comme s'ils avaient plus appris (et évalués en conséquence). Alors que les pistes explicatives précédentes se réfèrent à la théorie de la socialisation, cette troisième se rapporte plutôt à des théories du type labelling, mettant au cœur de l'analyse la manière dont les individus sont jugés, mais aussi la façon dont ils classent et catégorisent les actions et les personnes. Des effets institutionnels de ce type sont d'autant plus probables que le placement dans un groupe est public, et relativement stable.

Certaines recherches font effectivement apparaître ces processus d'étiquetage (1), notamment dans les décisions que les enseignants ont à prendre concernant les élèves : au-delà des résultats «objectifs » de ces derniers, la représentation qu'ils ont de leur compétence (et le pronostic sur l'avenir souvent implicite dans les décisions à prendre) s'ancre sur des informations telles que l'assignation à un groupe, le niveau supposé de l'école, etc. Mingat (1991) observe par exemple, dans une recherche sur les GAPP (structure de rééducation précoce), qu'en fin d'année (toutes choses égales par ailleurs) un élève qui a été rééduqué se voit plus souvent proposer un redoublement.

Soulignons que ces attentes spécifiques des enseignants vont interagir de manière dynamique avec les représentations des élèves, dans les différents groupes de niveau. On sait en effet que les élèves perçoivent d'autant mieux les attentes réelles des enseignants à leur encontre qu'ils sont placés dans tel ou tel groupe à la signification patente. Or le rôle spécifique des attentes des maîtres dans la dynamique pédagogique est bien connu (Rosenthal, Jacobson, 1972, et la notion d' «effet Pygmalion», à savoir le fait que la prédiction par le maître des performances de l'élève tend à influencer non seulement l'évaluation qu'il en fait, mais, plus paradoxalement, ces performances elles-mêmes). Au total, les classes de niveau, qui constituent donc des «milieux»d'apprentissage et de socialisation étiquetés dès le départ, fonctionneraient largement sur le mode de la prophétie qui se réalise d'elle-même.

(1) Développée par les spécialistes de la déviance, la notion d'étiquetage (labelling) insiste sur le fait que c'est la stigmatisation elle-même qui crée les comportements d'échec ou de déviance, en engendrant une dynamique d'attentes et d'autodévalorisation qui tend à s'entretenir d'elle-même. 
Il reste à évaluer, parmi les processus variés susceptibles de participer à la genèse des effets pervers des groupes de niveau, celui ou ceux qui s'avéreraient les plus importants. Une recherche récente (Pallas et al., 1994) montre que l'affectation à un groupe a bien un effet sur le test final, la progression étant meilleure dans les groupes de haut niveau; on remarque que cette meilleure progression, bien que ce soit des groupes de niveau en lecture qui aient été constitués, vaut à la fois pour la lecture et les mathématiques, ce qui signifierait soit que les élèves des meilleurs groupes apprennent à apprendre mieux en général (et pas seulement en lecture), soit que l'assignation à un groupe fort accroît la motivation à apprendre en général.

Par contre, on ne trouve pas trace d'effet systématique de l'assignation à un groupe sur l'estime de soi des élèves. Tout au plus certains travaux montrent-ils une amélioration de l'image de soi chez les élèves (et une détérioration chez les élèves forts) placés dans des classes homogènes. Enfin, des effets «institutionnels» sont décelés : les parents comme les enseignants considèrent comme plus compétents les élèves des groupes forts, et font des pronostics plus -optimistes pour leur avenir. Cela dit, lorsque l'on raisonne à résultats scolaires identiques, cet effet institutionnel se réduit : une partie (les trois quarts pour les maîtres, les deux cinquièmes pour les parents) de ce différentiel d'attentes correspond au fait qu'effectivement ces enfants ont plus appris ; mais les éducateurs réagissent néanmoins différemment aux performances effectives des élèves selon leur groupe.

$\mathrm{Au}$ total, les modifications pédagogiques induites par l'organisation en classes de niveau pèsent donc d'un poids plutôt plus fort que les mécanismes de nature psychosociale (effets institutionnels, effets d'attente, normes de groupe). Soulignons pour finir la richesse de ce champ de recherche où s'illustre particulièrement bien la complémentarité entre d'une part des approches. «macro» dégageant les effets des groupes de niveau et tentant d'évaluer leur rôle dans la genèse des inégalités sociales de scolarisation, et d'autre part des travaux «micro» cherchant à comprendre les processus qui engendrent les effets constatés (Gamoran, Berends, 1987).

Par rapport à cet état des connaissances sur le sujet, ce travail vise à apporter des informations empiriques nouvelles - dans le contexte français - et au niveau du collège. Si, en effet, des analyses nombreuses existent sur l'influence des caractéristiques personnelles des élèves et sur le contexte global de l'établissement (voir notamment Bressoux, 1994; Cousin, 1993 ; Duru-Bellat, Mingat, 1988), l'étude de la constitution des groupes d'élèves et de leurs conséquences sur les progressions et les carrières est plus lacunaire. Le travail est présenté en trois étapes successiives : la première examine la question de la constitution des classes de niveau en insistant sur le travail incontournable de construction de l'objet «classe de niveau »; la seconde s'attache à l'identification des effets du contexte classe sur les progressions des élèves en cours de cycle et la troisième à ses effets sur l'orientation. 


\section{Opérationnaliser la pratique des classes de niveau et en évaluer la fréquence au niveau collège}

\section{Une pratique dont l'observation demande une construction spécifique}

Il est clair que l'on ne peut dire de façon abrupte que tel ou tel collège constitue des classes de niveau. Sans parler du caractère naïvement dichotomique d'une telle assertion, la définition de ce concept «constitution de classes de niveau» ne va pas de soi et repose sur la manière conventionnelle dont on a décidé de l'opérationaliser. Il apparaît a priori pertinent d'imaginer que cette pratique est mise en œuvre à des degrés variés sachant que la constitution de classes de niveau peut se lire dans une double dimension à savoir: 1) différence dans le niveau moyen des classes d'un même établissement; et 2) modification (réduction) de l'hétérogénéité au sein des différentes classes. En termes d'analyse de variance, faire des classes de niveau ce serait donc partitionner la variance initiale du niveau individuel des élèves du collège en une variance intergroupe (différence de niveau moyen d'une classe à l'autre) et une variance intragroupe caractérisant les disparités de niveau des élèves de chaque classe; à variance initiale donnée, plus les classes diffèrent en niveau plus chacune d'entre elles aura tendance en moyenne à avoir un public de niveau plus homogène.

Si l'on raisonne sur l'ensemble des établissements, l'analyse de la politique de répartition des élèves entre les différentes divisions peut être conduite à partir de l'estimation de la proportion d'établissements pour lesquels on peut raisonnablement émettre l'hypothèse d'une répartition aléatoire des élèves, et réciproquement la représentation des collèges qui composent des classes de niveau (2). Seuls ont été considérés les collèges où le nombre de divisions est assez important pour qu'il soit légitime d'étudier la ventilation des élèves entre les différentes divisions. Nous avons retenu ici les établissements en comptant au moins quatre. Selon ce critère, nécessairement arbitraire, seulement près de $20 \%$ répartissent leurs élèves de façon à respecter une hétérogénéité intraclasse. Complémentairement, $80 \%$ des établissements réaliseraient de fait des classes de niveau, sachant que certaines d'entre elles utilisent entre autres le système des cycles aménagés qui constituent de façon manifeste au moins une «classe de niveau». Il convient de rappeler que ce chiffre est établi sur

(2) Cette classification repose sur l'examen simultané des rapports de Fisher estimés sur le score global des tests à l'entrée de la sixième, et du $\mathbf{R}^{2}$ fourni par l'analyse de la variance menée sur chaque collège. L'hypo- thèse de répartition aléatoire des élèves dans les divisions a été rejetée pour tout collège pour lequel le rapport $F$ atteint une valeur correspondant à un seuil de probabilité inférieur à $10 \%$. 
l'échantillon DEP dont nous avons souligné plus haut les particularités; cela dit, la surestimation éventuelle est sans doute modérée car, nous le verrons ultérieurement, les collèges populaires n'ont qu'une tendance discrète à constituer plus souvent des classes de niveau.

Une démarche alternative pour caractériser la situation de chacun des collèges consiste à repérer deux pratiques extrêmes entre lesquelles prendraient place les pratiques effectives des collèges : à un bout du spectre, une pratique que l'on pourrait qualifier de «degré zéro de classes de niveau » consisterait à répartir les élèves du collège dans des classes dont le niveau moyen et le degré d'hétérogénéité seraient identiques et égaux à la valeur moyenne de ces indicateurs pour la population d'ensemble du collège, comme l'illustre le collège $A$ dans la Figure $I$. À l'autre bout du spectre, une pratique maximale de constitution de classes de niveau serait fondée sur un classement préalable de tous les élèves du collège par ordre croissant ou décroissant selon leur niveau initial et sur une affectation ordonnée de ces élèves dans les différentes classes sur cette base ; on constituerait ainsi des classes dont le niveau moyen serait strictement hiérarchisé et dont l'hétérogénéité à l'intérieur de chacune d'entre elles serait minimale; le collège $B$ dans le schéma ci-après va dans cette direction.

FIGURE I. - Deux exemples opposés de pratique de constitution de classes dans un collège

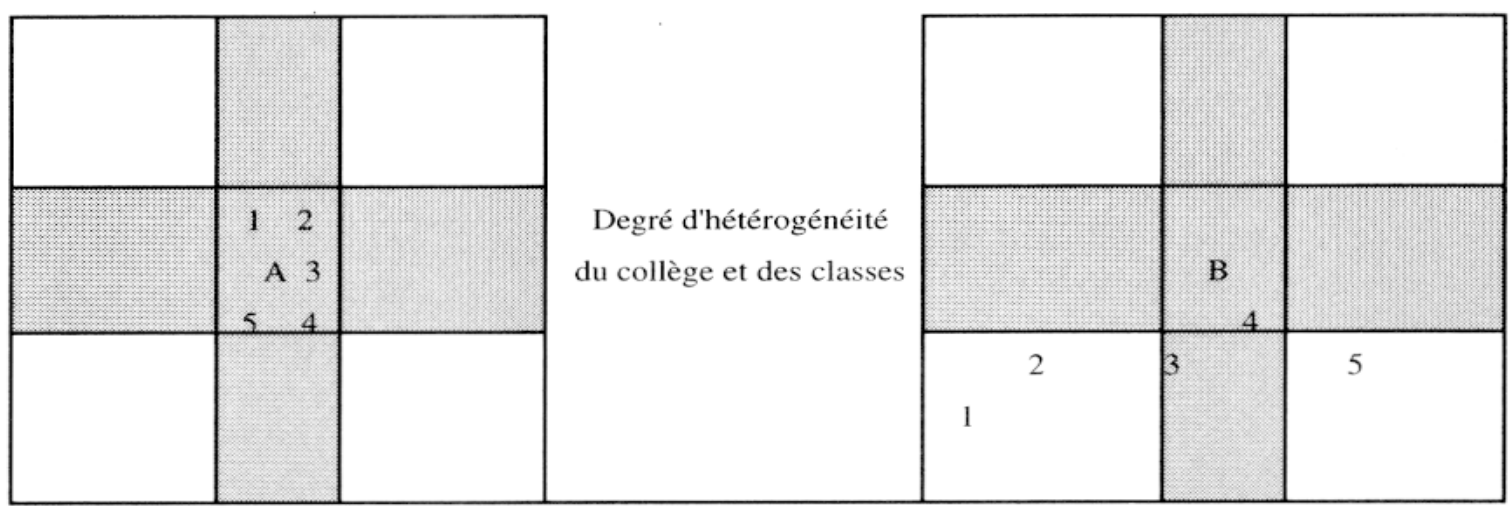

Niveau moyen du colège et des classes

Niveau moyen du colège et des classes

Même s'il est clair que la pratique de chacun des collèges s'inscrit sur un continuum, il importe de fixer des seuils si l'on entend dénombrer les collèges qui feraient, ou ne feraient pas, de classes de niveau. Pour ce faire, il convient d'identifier un seuil au-delà duquel on considérera qu'une classe s'écarte significativement de la situation moyenne de l'établissement; ce seuil comporte bien évidemment une partie d'arbitraire. On a considéré ici que si la moyenne d'une classe s'écartait de plus de deux points dans l'échelle des acquisitions (moyenne 100, écart-type 15, sur les données individuelles), alors cette classe présentait un écart suffisant par 
rapport à la situation pour être classée comme une classe de niveau. Compte tenu du caractère conventionnel de ces deux points de distance (en plus ou en moins), une définition alternative s'est fondée sur un écart de trois points. On identifie ainsi les classes extérieures à la «bande» définie par ces deux bornes, et un collège a une pratique d'autant plus intense de constitution de classes de niveau qu'une proportion importante de ses classes est extérieure à cette bande. On fait de même pour l'hétérogénéité des classes et l'on considère que l'on a constitué une classe homogène lorsque l'écart-type de la classe est inférieur de deux ou trois points de l'écart-type associé à la distribution du niveau individuel de l'ensemble de la population du collège.

Dans la mesure où le nombre de classes diffère d'un collège à l'autre, au lieu de compter le nombre de classes qui dévient significativement de la moyenne du collège, il apparaît préférable de calculer un indicateur qui rapporte ce nombre au nombre total de classes de l'établissement. Cet indicateur peut être calculé sur la base du niveau moyen des élèves et sur celle de l'hétérogénéité de ce niveau sachant que si ces deux indicateurs ont une tendance «mécanique » à se ressembler, il existe toutefois des différences entre eux; c'est pourquoi il est apparu préférable de calculer leur somme pour définir un premier indicateur synthétique de l'intensité de la pratique de constitution de classes de niveau au sein des différents collèges de l'échantillon.

Selon les deux seuils retenus, il y aurait entre 45 et $57 \%$ des classes de collèges dont le public se distinguerait significativement des caractéristiques moyennes des élèves du collège par le niveau moyen et/ou l'hétérogénéité. Quand on porte l'examen au niveau de l'établissement, on peut calculer la proportion des classes, au sein de chaque collège, qui s'écarterit de manière notable des paramètres moyens du collège (plus de deux ou trois points dans la dimension du niveau moyen d'acquis des élèves ou de deux ou trois points dans celle de l'hétérogénéité de ce niveau au sein de la classe). Ces proportions varient de façon sensible d'un collège à l'autre.

Soulignons que l'identification de la proportion des collèges dont on pourrait dire qu' «ils réalisent des classes de niveau » procède aussi d'un nécessaire arbitraire, non seulement dans le choix des indicateurs ci-dessus (deux, trois ou $x$ points dans telle et/ou telle des deux dimensions considérées), mais aussi dans le choix du pourcentage des classes à partir duquel on décide que tel collège adopte la pratique générique de «constitution de classes de niveau» (3). Compte tenu de ceci, il apparaît préférable de présenter des fourchettes plutôt qu'une valeur unique qui pour être «précise» n'en serait pas moins essentiellement conventionnelle. Au total, ces

(3) Il peut certes exister des collèges qui constituent une seule classe spécifique très typée, soit dans des établissements populaires pour constituer une bonne classe visant à re- tenir les élèves les plus brillants, soit dans les collèges «chics» pour constituer une classe regroupant les élèves plus difficiles. 
différents choix conduisent à estimer que, sur la base du seuil de deux points, il $\mathrm{y}$ aurait entre 15 et $32 \%$ des collèges qui auraient une pratique de constitution de classes de niveau relativement modérée, alors qu'entre 26 et $51 \%$ des établissements constitueraient leurs classes selon une structure clairement hiérarchisée.

S'il est nécessaire de se donner des seuils pour produire une réponse catégorielle (tel établissement fait ou non des classes de niveau), il est souhaitable d'éliminer cette contrainte si l'on veut estimer l'intensité des pratiques de constitution de classes de niveau d'un établissement et en tester l'importance dans un modèle rendant compte des acquisitions et des carrières des élèves en fonction de leur contexte de scolarisation. On peut alors construire un indicateur synthétique mesurant la distance moyenne existant entre le point caractérisant le public moyen du collège (niveau moyen des élèves et hétérogénéité de ce niveau) et les points caractéristiques de chaque classe dans ces deux dimensions. Cet indicateur présente l'avantage de ne pas opérer pour chaque classe une affectation dichotomique entre classe de niveau ou non, mais d'utiliser un continuum distinctif entre classes selon leur degré d'éloignement des caractéristiques de leur public par rapport à celles de l'établissement. Dans la mesure où les deux dimensions de l'espace considéré ont comme mesure commune des points sur l'échelle d'évaluation retenue (moyenne 100, écart-type 15), on peut calculer directement la distance rectilinéaire entre le point moyen (respectivement niveau moyen $\mathrm{N}$ et hétérogénéité moyenne $\mathrm{H}$ ) et chacun des points caractéristiques de chaque classe $\mathrm{j}$ (respectivement $\mathrm{N}_{\mathrm{j}}$ et $\mathrm{H}_{\mathrm{j}}$ ); il suffit ensuite de calculer la moyenne de cette distance entre les $n$ différentes classes d'un même établissement pour obtenir l'indicateur synthétique recherché (dont la valeur moyenne est de 7,78 points et l'écart-type de 3,47 points).

$$
D=1 / n \operatorname{Som}_{j=1, n}\left[\operatorname{Abs}\left(N-N_{j}\right)+\operatorname{Abs}\left(H-H_{j}\right)\right]
$$

Les deux indicateurs précédemment construits entretiennent à l'évidence des relations statistiques puisqu'ils sont deux façons de lire une même réalité; la variance commune entre les deux types d'indicateur est de l'ordre de 0,80 . Selon les traitements, nous utiliserons l'un ou l'autre de ces deux indicateurs.

Une question connexe à celle de la constitution de classes de niveau est d'identifier ses conséquences sur la tonalité sociale des classes ainsi constituées. Dans quelle mesure les classes de niveau reviennent-elles de façon jointe à typer socialement les différentes classes d'un établissement? On peut en faire l'hypothèse dans la mesure où le niveau des élèves est partiellement lié à leur origine sociale, sachant qu'en outre il peut exister des biais sociaux complémentaires dans l'affectation des élèves dans les classes au-delà de ce qui tient à leur niveau scolaire personnel. Pour tester cette hypothèse, nous avons en premier lieu construit un indicateur mesu- 
rant l'ampleur des écarts de tonalité sociale entre les classes d'un même établissement.

Si au sein d'un collège, tous les élèves étaient répartis indépendamment de leur origine sociale entre les différentes classes, le pourcentage d'élèves de milieu défavorisé/favorisé serait identique dans toutes les classes et égal à la valeur moyenne de cette statistique dans le collège (qui diffère évidemment d'un collège à l'autre). Plus les classes sont socialement typées au sein d'un établissement, plus la proportion d'élèves défavorisés/favorisés au sein de chacune d'entre elles s'écarte de la valeur moyenne du collège. Pour construire un indicateur de cette disparité sociale des classes, on peut calculer la moyenne de cet écart sur l'ensemble des classes en prenant soin de prendre en compte la valeur absolue de ces écarts (pour éviter l'effet de compensation inhérent à la nécessaire symétrie entre les différenciations sociales des différentes classes d'un même établissement). Alors que dans certains collèges cet indicateur est faible (voire même nul), on observe que d'autres collèges réalisent des classes socialement très différenciées.

On note alors que ces variations d'un collège à l'autre dans la constitution de classes socialement différenciées ne sont que très faiblement liées à la pratique de constitution de classes de niveau au sein de l'établissement. La part de variance de l'indicateur de différenciation sociale des classes associée à cette pratique n'est que $2 \%$. La relation est toutefois positive et statistiquement significative; la constitution de classes de niveau ayant donc plutôt tendance à constituer des classes plus typées socialement. Cela dit, l'analyse montre que les collèges qui constituent des classes socialement typées le font essentiellement par d'autres voies, notamment par l'intermédiaire du groupement d'élèves selon la langue vivante, comme nous le verrons par la suite, au-delà de ce qui tient à la valeur scolaire des élèves.

Quels collèges constituent, et avec quelle intensité, des classes de niveau?

Enfin, l'opérationalisation de la notion de «pratique de classes de niveau dans un établissement », par les indicateurs constitués plus haut, permet de déterminer les caractéristiques du collège qui s'avèrent associées à ces pratiques. Les analyses multivariées qui ont été réalisées montrent tout d'abord que les variables structurelles caractérisant le public d'élèves n'exercent au total qu'une influence limitée sur la variabilité des pratiques de constitution de classes de niveau. En effet, le pourcentage de variance expliquée varie, selon les modèles (et selon que l'on intègre ou non les collèges ayant mis en place un cycle aménagé) de 15 à $31 \%$.

On observe par ailleurs que le niveau moyen des élèves du collège est associé positivement à la pratique de classes de niveau : cette pratique est d'autant plus intense que les élèves ont en moyenne un niveau initial fort. Ce résultat peut apparaître surprenant dans la mesure où le discours des 
acteurs a tendance à mêler la notion d'hétérogénéité avec la faiblesse moyenne du niveau des élèves. Le degré d'hétérogénéité par contre joue bien dans le sens attendu : un collège a d'autant plus tendance à constituer des classes de niveau que l'hétérogénéité de son public est élevée. Si l'on tient compte de l'écart-type de la distribution de ces deux variables $(5,5$ pour le niveau moyen, 1,7 pour la mesure de l'hétérogénéité), on peut estimer que de façon globale l'influence statistique du degré d'hétérogénéité du public d'élèves est environ entre une et demie et deux fois plus forte que celle (positive) du niveau moyen.

En ce qui concerne l'effet de la tonalité sociale du collège sur l'intensité de la pratique de classes de niveau, les résultats indiquent une légère tendance à une pratique plus intense quand le public du collège est plus défavorisé. Il ne s'agit toutefois que d'une tendance eu égard d'une part à la faible significativité du coefficient et d'autre part à sa relative faiblesse numérique (en tenant compte des écart-types respectifs, on peut estimer que l'impact de la tonalité sociale est entre trois et quatre fois inférieur à celui de l'indicateur d'hétérogénéité). Enfin, la taille du collège dont on aurait pu penser qu'elle serait positivement associée à la pratique des classes de niveau (il est a priori plus commode de constituer des classes de niveau dans un ensemble plus vaste d'élèves) n'exerce pas d'influence nette si ce n'est sur le sous-échantillon des collèges ne constituant pas de cycle aménagé, où la variable joue dans le sens attendu.

Au total, on peut donc conclure à une influence finalement assez limitée des caractéristiques scolaires et sociales du public des élèves d'un collège sur ses pratiques de constitution de classes (de niveau et de type aménagé). À l'évidence, d'autres facteurs non intégrés dans l'analyse exercent une influence (les caractéristiques des enseignants, le projet d'établissement, la personnalité du chef d'établissement, etc. sont d'éventuels candidats). En référence à ces variables, on peut donc conclure à un assez fort degré d'aléa dans les pratiques de constitution de classes. Les acteurs locaux exercent donc de fait une marge de manœuvre non négligeable, choisissant ou non de créer de la variété entre leurs classes au-delà de l'hétérogénéité dont ils «héritent» en raison de la carte scolaire.

Pour analyser plus précisément ce point, une stratégie consiste à désagréger la variance globale du niveau initial des élèves en trois composantes : 1) ce qui se joue entre établissements par le fait qu'ils accueillent des élèves de niveau différent ; 2) ce qui se joue au sein des établissements par la constitution de classes de niveau plus ou moins différent; et 3 ) ce qui reste comme variabilité entre élèves au sein des classes ainsi constituées. Le Tableau I donne la décomposition (en pourcentage) de la variance totale.

Il apparaît en premier lieu que les différences de public d'un établissement à l'autre n'expliquent que $13,3 \%$ de la variance globale. Ce chiffre est relativement faible par rapport à l'intuition commune qui considère les collèges comme très différents en raison de la carte scolaire. Toutefois, 
Tableau I. - Décomposition de la variance totale du score initial des élèves

\begin{tabular}{|c|c|c|c|}
\hline Variance Totale & $\begin{array}{c}\text { Variance inter- } \\
\text { établissements }\end{array}$ & $\begin{array}{c}\text { Variance intra-établissement } \\
\text { et inter-classes }\end{array}$ & Variance intra-classes \\
\hline $100 \%$ & $13,3 \%$ & $21,4 \%$ & $65,3 \%$ \\
\hline
\end{tabular}

ce chiffre apparaît faible surtout par comparaison avec ce qui se joue au sein des établissements dans la constitution de leurs classes : en effet, la part de la variance intra-établissements et inter-classes représente $21,4 \%$ de la variance totale; en d'autres termes, les établissements «fabriquent» davantage de variance inter-classes qu'ils n'en «subissent» par la carte scolaire. Alors que l'on dénonce souvent les limites à la politique éducative imposée par les contraintes de la carte scolaire, ces chiffres soulignent que les pratiques effectives des établissements quand ils constituent leurs classes ont de fait des conséquences plus fortes en ce qu'elles contribuent plus à la segmentation des élèves par niveau. Il reste possible que l'importance de la variabilité inter-établissements soit ici un peu minorée compte tenu d'une certaine spécificité de l'échantillon (plus populaire et un peu moins hétérogène que la population totale des collèges français); cela dit, les ordres de grandeur relatifs de ce qui est imposé aux établissements/de ce qu'ils en font, attestent du poids des pratiques internes. Il reste maintenant à examiner dans quelle mesure ces pratiques ont des conséquences visibles sur les élèves, sachant que nous nous intéresserons dans ce texte uniquement aux acquis scolaires et aux orientations prises.

\section{Les progressions des élèves, dans des classes de niveau et d'hétérogénéité variés}

Nous nous attacherons ici essentiellement à l'influence des variables de contexte, sachant que celle-ci ne peut être évaluée qu'à la marge d'une modélisation plus globale prenant en compte également les caractéristiques individuelles des élèves; ces données ont alors le statut de variables de contrôle et ne feront pas ici l'objet d'un commentaire détaillé.

Partons des modèles construits pour tenter d'expliquer les progressions des élèves, entre l'entrée en $6^{e}$ et la fin de la $5^{e}$ (sachant qu'il s'agit de la seconde $5^{\mathrm{e}}$ pour les élèves ayant redoublé cette classe), sur la base des tests de connaissances administrés aux élèves de l'échantillon à ces deux périodes (ces scores ont été standardisés avec une moyenne de 100 et un écart-type de 15) (4). Dans un premier temps (modèles 1 et 2, Tableau III),

(4) C'est par rapport à ces références, et notamment à l'écart-type de 15, que doivent s'interpréter les effets des variables concernant la progression des élèves. 
l'impact des caractéristiques personnelles des élèves a été estimé; il s'avère élevé ( $52 \%$ de la variance du score de fin de $5^{\mathrm{e}}$ ), mais essentiellement du fait de la prise en compte du niveau initial. Dès lors que l'on travaille sur les progressions, l'effet de l'appartenance sociale apparaît assez modéré : sur la base d'une première analyse prenant en compte la profession du père de manière détaillée, deux groupes relativement homogènes en regard de cette analyse ont été distingués, à savoir d'une part les enfants de père cadre, profession intermédiaire ou agriculteur, d'autre part les autres catégories (ouvrier, employé, inactif (5),..); seul un peu plus d'un point supplémentaire distingue les progressions du premier groupe par rapport au second. On observe aussi de meilleures progressions quand la mère est cadre (supérieur ou moyen), les enfants de mères moins qualifiées ne se démarquant pas de ceux dont la mère est au foyer.

L'âge exerce un effet plus marqué ( $-3,6$ points par année d'âge en moyenne), et le sexe joue significativement mais avec une ampleur limitée ( $-0,6$ point pour les garçons). On remarque également l'impact significatif de la variable nationalité de l'élève, avec une meilleure progression des élèves de nationalité étrangère $(+1$ point), sachant que lorsque l'origine précise de l'enfant est prise en compte, ce sont les élèves d'origine maghrébine qui font preuve des progressions les plus marquées $(+2$ points par rapport aux élèves français de caractéristiques comparables). Toujours est-il qu'il y a donc place, entre la $6^{\mathrm{e}}$ et la fin de la $5^{\mathrm{e}}$, pour des facteurs autres, susceptibles de participer à la genèse de progressions plus ou moins fortes.

Centrons-nous maintenant sur l'influence éventuelle des modes de groupement d'élèves sur les progressions en cours de cycle; on examinera en premier lieu les différences existant de fait entre les classes quant à leur niveau moyen et celui de leur hétérogénéité pour aborder ensuite l'influence spécifique de mesures institutionnelles telles que le regroupement de certains élèves dans des cycles en trois ans. Enfin, nous nous demanderons dans quelle mesure des classes différenciées sont constituées de fait par le truchement de la langue vivante étudiée, avec quelle incidence en termes de progression.

\section{L'influence des classes de niveau sur les progressions des élèves}

Pour appréhender l'effet éventuel des classes de niveau, pratique souvent implicite (sauf lorsqu'elle prend la forme drastique du cycle aménagé), il convient de calculer pour chaque classe le niveau moyen des élèves à l'entrée dans le cycle ainsi que l'hétérogénéité du niveau des élèves

(5) Les modèles plus détaillés font apparaître en fait des progressions plutôt inférieures quand le père est inactif ou chômeur par rapport aux autres catégories « défavorisées ». 
autour de ce niveau moyen; ces données sont ensuite affectées à chaque élève de chaque classe. Notons que seuls sont connus dans l'enquête le niveau moyen et l'hétérogénéité de la classe de $6^{\mathrm{e}}$; on peut néanmoins faire l'hypothèse que dans la majorité des cas il n'y a pas de remaniements substantiels de la distribution des élèves entre les classes de $6^{\mathrm{e}}$ et $5^{\mathrm{e}}$.

La manière la plus directe d'examiner si des élèves de niveau initial comparable progressent plus ou moins selon le niveau de la classe où ils sont scolarisés consiste à faire un tableau croisant ces deux paramètres (Tableau II). Sur la base de ce tableau, il semble que les élèves progressent d'autant mieux qu'ils sont scolarisés dans une classe de niveau moyen élevé ; ainsi, 4,0 points séparent les élèves les plus faibles scolarisés dans les classes les plus faibles de ceux scolarisés dans les meilleures classes; chez les élèves de niveau initial élevé, l'écart est plus marqué puisqu'il atteint 7,1 points.

\begin{tabular}{|c|c|c|c|c|c|}
\hline & \multicolumn{5}{|c|}{ Niveau individuel en début de $6^{\mathrm{e}}$} \\
\hline & $<85$ & $85-95$ & $95-105$ & $105-115$ & $>115$ \\
\hline $\begin{array}{l}\text { Niveau moyen de la classe } \\
\cdot<90 \\
.90-96 \\
.96-103 \\
.103-108 \\
.>108\end{array}$ & $\begin{array}{l}82,6 \\
84,6 \\
85,2 \\
86,7 \\
86,6\end{array}$ & $\begin{array}{l}91,8 \\
93,6 \\
94,7 \\
94,6 \\
97,4\end{array}$ & $\begin{array}{r}96,7 \\
100,2 \\
100,9 \\
100,9 \\
103,1\end{array}$ & $\begin{array}{l}104,9 \\
106,6 \\
107,6 \\
108,2 \\
109,8\end{array}$ & $\begin{array}{l}109,9 \\
115,6 \\
115,2 \\
116,4 \\
117,0\end{array}$ \\
\hline
\end{tabular}

Il convient toutefois de prendre ces premiers résultats avec précaution, et ce pour deux raisons complémentaires :

- La première est que d'autres facteurs (sociaux notamment) peuvent affecter les progressions des élèves; dans la mesure où ils sont inégalement distribués dans les différents groupes constitués dans le Tableau II, ceci peut entraîner des artefacts.

- La seconde raison est que le tableau utilise des groupes dont le score initial se situe sur une plage de variation, qui pour être réduite par la catégorisation elle-même n'en est pas pour autant nulle. Il s'ensuit qu'au sein d'une catégorie de niveau initial individuel donnée, la distribution des élèves selon ce critère est nécessairement décalée selon le niveau de la classe; ceci conduit à surestimer l'effet du niveau moyen de la classe sur les progressions individuelles.

L'existence de ces deux problèmes milite pour l'utilisation de modèles multivariés qui, d'une part prennent en compte simultanément l'ensemble des facteurs et, d'autre part et surtout, permettent de contrôler le niveau initial des élèves. Dans la mesure où les données disponibles concernant le niveau initial des élèves ne sont pas d'une fiabilité ou d'une fidélité 
parfaites (des épreuves de ce type ont généralement un coefficient de fidélité compris entre 0,85 et 0,90 ), on est exposé à l'influence éventuelle d'erreurs de mesures (6). Celles-ci peuvent conduire à sous-estimer l'intensité de l'influence du niveau initial dans le modèle de progression (avec en outre des conséquences non désirables sur l'estimation de l'effet des classes de niveau, dans la mesure où les élèves n'y sont pas répartis aléatoirement en fonction de leur score initial). Une façon de contourner cette difficulté consiste à utiliser un modèle de mesure (par exemple LISREL) pour utiliser un score estimé plutôt qu'un score brut; ici nous avons considéré que la prise en compte d'une moyenne faite sur la base de 8 sub-tests dans deux matières conduisait à réduire ces aléas et que les résultats obtenus ainsi seraient largement exempts de biais.

Par ailleurs, dans la mesure où l'on s'intéresse ici à l'articulation de variables qui se situent à des niveaux d'agrégation différents (les progressions individuelles des élèves selon les caractéristiques de leur classe), il pourrait être pertinent d'inscrire ces estimations à l'intérieur d'une structure multiniveau (voir Bryk, Raudenbush, 1992). Cela dit, une analyse comparative des résultats obtenus selon le modèle multiniveau et le modèle standard (Bressoux et al., 1997) conclut que les modèles individuels mono-

TABLEAU III. - Facteurs individuels et contextuels de la progression des élèves en cours de cycle

\begin{tabular}{|c|c|c|c|c|}
\hline & Modèle 1 & Modèle 2 & Modèle 3 & Modèle 4 \\
\hline Constante & 27,1 & 77,0 & 68,6 & 79,2 \\
\hline $\begin{array}{l}\text { Score de début de } 6^{\mathrm{e}} \\
\text { Étranger/Français } \\
\text { Âge à l'entrée en } 6^{\mathrm{e}} \\
\text { Père «favorisé »/« défavorisé » } \\
\text { Mère cadre/autre } \\
\text { Garçon/fille } \\
\text { Niveau classe en } 6^{\mathrm{e}} \\
\text { Hétérogénéité classe en } 6^{\mathrm{e}} \\
\text { GC1/GC5* } \\
\text { GC2/GC5 } \\
\text { GC3/GC5* } \\
\text { GC4/GC5* } \\
\text { Position classe/collège }\end{array}$ & $+0,74(* * *)$ & $\begin{array}{l}+0,65(* * *) \\
+1,1 \quad(* * *) \\
-3,6 \quad(* * *) \\
+1,1 \quad(* * *) \\
+1,1 \quad(* * *) \\
-0,6 \quad(* * *)\end{array}$ & $\begin{array}{l}+0,62(* * *) \\
+1,1 \quad(* * *) \\
-3,6 \quad(* * *) \\
+1,0 \quad(* * *) \\
+1,0 \quad(* * *) \\
-0,6 \quad(* * *) \\
+0,10(* * *) \\
+0,08(* * *)\end{array}$ & $\begin{array}{l}+0,62(* * *) \\
+1,1 \quad(* * *) \\
-3,6 \quad(* * *) \\
+1,0 \quad(* * *) \\
+1,1 \quad(* * *) \\
-0,6 \quad(* * *)\end{array}$ \\
\hline$\%$ de variance expliquée; $\mathrm{R}^{2}$ & 49,6 & 52,2 & 52,4 & 52,4 \\
\hline
\end{tabular}

* GC1 classe faible à hétérogénéité faible

GC2 classe faible à hétérogénéité moyenne ou forte

GC3 classe moyenne à hétérogénéité faible

GC4 classe moyenne à hétérogénéité moyenne ou forte

GC5 classe forte à hétérogénéité faible, moyenne ou forte

(6) Pour une bonne discussion sur ce point, voir Davidson et MacKinnon (1993). 
niveau donnent des ordres de grandeur fiables pour un coût de traitement bien inférieur, compromis acceptable lorsque l'on centre l'analyse sur l'efficacité et non sur l'équité; pour cette raison, nous avons conservé ici l'approche classique d'estimation.

Dans un premier modèle (modèle 3 , Tableau III), le niveau moyen et l'hétérogénéité de la classe fréquentée ont été ajoutés aux variables individuelles. Si le pouvoir explicatif du modèle 3 ne s'accroît que peu par rapport à celui du modèle 2 (mais les variables individuelles n'ont globalement qu'une influence mesurée à $2,6 \%$ de la variance), le niveau moyen de la classe a tendance à affecter néanmoins positivement les progressions, celles-ci étant donc d'autant meilleures que la classe est de niveau moyen élevé; sachant que l'écart-type de cette variable est de 8,3 points et que son coefficient dans le modèle 3 est égal à 0,10 , cela implique que la variabilité dans le niveau moyen de la classe se traduit par de notables écarts dans le niveau atteint à la fin du cycle (7): un élève de niveau initial donné scolarisé dans une bonne classe (112 de niveau initial) réalise une progression de 2 points supérieure à celle que réaliserait son homologue scolarisé dans une classe faible (92 de score initial); encore ne s'agit-il pas de cas très contrastés puisque la plage de la distribution du niveau moyen des classes est de 50 points.

De manière plus inattendue, l'hétérogénéité est aussi associée positivement (et linéairement) à de meilleures progressions; mais la relation est également moins intense, d'une part en raison de la faiblesse du coefficient, et d'autre part du fait que l'écart-type de l'indicateur mesurant le degré d'hétérogénéité est plus faible (2,95 points) (8); cela dit, on progresserait tout de même d'autant moins bien que la classe fréquentée est d'un niveau plus homogène.

Pour mieux comprendre l'articulation de ces deux paramètres, nous avons examiné la distribution des élèves selon le niveau moyen et le degré d'hétérogénéité de leur classe et construit le Tableau IV qui permet de distinguer 9 groupes (groupes notés 11 à 33 ).

Un premier modèle instrumental montre que le type de classe fréquentée (au sens des 9 groupes précédemment définis) affecte significativement les progressions, celles-ci étant meilleures dans le groupe 31 , pris comme référence (les classes homogènes fortes), mais également de manière générale dans les classes de niveau élevé, quel que soit leur degré d'hétérogénéité (groupes 32 et 33 ); les progressions ne sont pas très différentes

(7) En utilisant une forme quadratique pour représenter l'influence du niveau moyen de la classe sur les progressions, on a pu conclure que la forme linéaire simple était adaptée : l'effet marginal de cette variable est donc globalement identique quel que soit son niveau, sans effet de seuil ni d'effet de plafond.

(8) En prenant en compte à la fois la différence dans la valeur des coefficients et dans l'écart-type des deux variables, on peut estimer que l'influence du niveau moyen de la classe est environ 3,5 fois plus élevée que celle du degré d'hétérogénéité. 
Revue française de sociologie

TABLEAU IV. - Effectifs des élèves selon le niveau moyen et le degré d'hétérogénéité de leur classe

\begin{tabular}{|l|c|c|c|}
\cline { 2 - 4 } \multicolumn{1}{l|}{} & $\begin{array}{c}\text { Hétérogénéité faible } \\
(<10)\end{array}$ & $\begin{array}{c}\text { Hétérogénéité moyenne } \\
(10-14)\end{array}$ & $\begin{array}{c}\text { Hétérogénéité forte } \\
(>14)\end{array}$ \\
\hline $\begin{array}{l}\text { Niveau moyen faible } \\
(<93)\end{array}$ & 598 (groupe 11) & 1302 (groupe 12) & 1156 (groupe 13) \\
$\begin{array}{l}\text { Niveau moyen moyen } \\
(93-105)\end{array}$ & 1316 (groupe 21) & 5707 (groupe 22) & 3760 (groupe 23) \\
$\begin{array}{l}\text { Niveau moyen élevé } \\
(>105)\end{array}$ & 2548 (groupe 31) & 2611 (groupe 32) & 401 (groupe 33) \\
\hline
\end{tabular}

dans les classes moyennes hétérogènes (groupes 22 et 23); elles apparaissent par contre significativement plus faibles dans les classes moyennes homogènes (groupe 21). On progresse encore moins dans les classes faibles, et ce d'autant plus qu'elles sont en outre homogènes (groupes 12 et 13 et surtout 11). S'il est globalement préférable d'être scolarisé dans une classe de bon niveau moyen, l'être dans une classe homogène constitue donc un handicap mais uniquement si la classe est de niveau moyen ou faible.

La Figure II illustre les différentiels de progression (en points) d'élèves comparables scolarisés dans ces différents types de classes, par référence aux élèves du groupe 31 (classes homogènes fortes).

\section{Figure II. - Différentiels de progression d'élèves comparables} selon le type de classe fréquentée

Écarts en points (réf groupe 31)

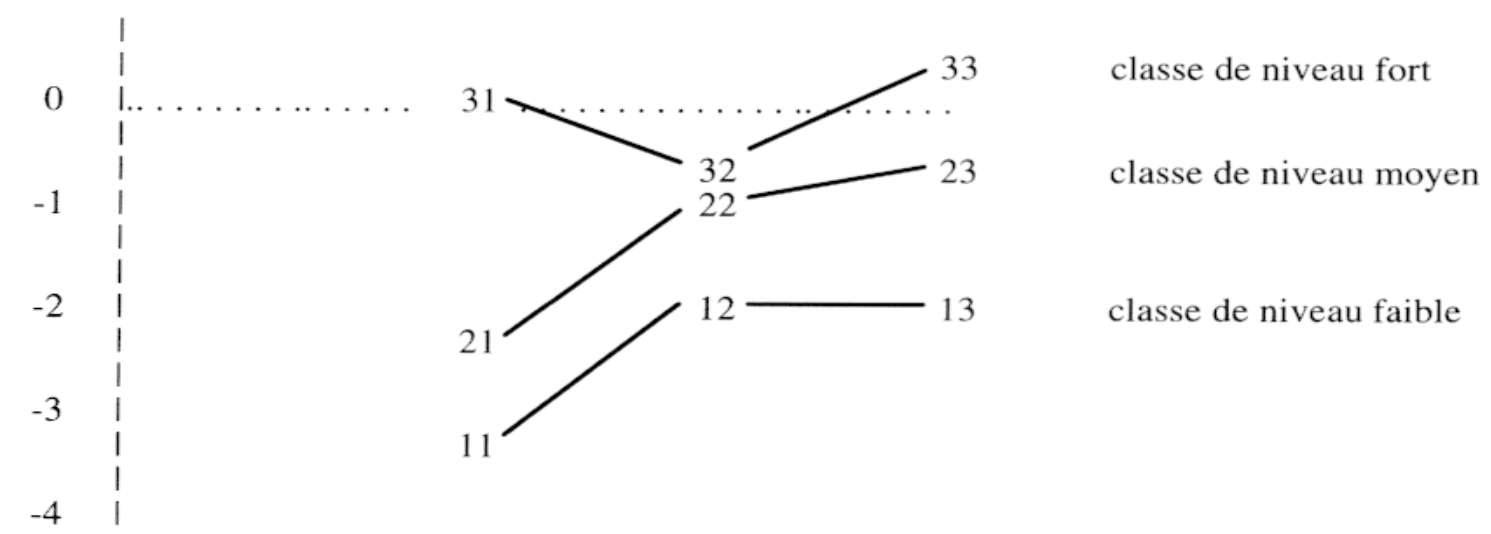

Degré d'hétérogénéité Faible Moyen Elevé 
Ces tendances ont amené à la construction d'une variable «type de classe de niveau regroupée » en 5 catégories : les classes de bon niveau moyen (au sein desquelles le niveau d'hétérogénéité est sans importance : variable (GC5), les classes moyennes à hétérogénéité moyenne ou forte (GC4), ou au contraire faible (GC3), et enfin les classes faibles à hétérogénéité moyenne ou forte (GC2) ou faible (GC1); ces variables sont utilisées dans le modèle 4). Il confirme que les progressions des élèves sont spécialement faibles dans les classes de niveau moyen à la fois faible et homogène.

Si donc la tendance générale à de plus faibles progressions dans les classes faibles, observée classiquement dans la littérature, se trouve ici confortée, il serait intéressant de tenter de faire la part des mécanismes de type instructionnel - moindre couverture des programmes, stimulation pédagogique moins intense,... - ou plutôt de type institutionnel - étiquetage négatif de la classe,... - susceptibles de produire ce type d'effets. Pour explorer de façon plus particulière la dimension instructionnelle, nous avons construit une variable caractérisant la position de l'élève par rapport au niveau moyen de sa classe, un élève de niveau donné pouvant ainsi se situer au-dessus ou en dessous du niveau moyen de cette dernière. Ceci permet d'évaluer si les progressions d'un élève donné sont affectées par sa position relative au sein de sa classe (position relative de l'élève = niveau initial de l'élève - niveau moyen de sa classe) : dans le cadre des modèles de progression du type de ceux présentés dans le Tableau III, on observe que le coefficient attaché à cette variable position est négatif et statistiquement très significatif; sa valeur numérique est de $-0,09$, impliquant qu'un élève situé à un écart-type du niveau moyen de sa classe voit sa progression en moyenne stimulée de $+1,05$ points s'il s'agit d'un élève faible en termes relatifs ou au contraire atténuée dans une proportion comparable s'il s'agit d'un élève situé au-dessus de la moyenne de sa classe. De façon globale, on est donc en présence d'un phénomène de régression à la moyenne : les élèves faibles profitent d'un niveau moyen supérieur au leur, alors que les élèves forts pâtissent de la fréquentation d'une classe d'un niveau moyen inférieur au leur.

Cela dit, compte tenu de la prise en compte d'une variable unique pour appréhender la position de l'élève dans sa classe, cette première mesure de l'effet de la variable position fait implicitement l'hypothèse d'un effet symétrique que l'élève soit faible ou fort en termes relatifs. Pour lever cette hypothèse implicite et autoriser une dissymétrie des effets, nous avons créé deux variables, précisant la position de l'élève selon qu'elle est en dessus ou en dessous du niveau moyen de la classe. Les résultats montrent le bien-fondé de cette distinction: les deux coefficients sont clairement (de façon statistiquement significative) différents, bien que la tendance générale de régression à la moyenne soit confirmée par le fait que les deux coefficients sont négatifs (respectivement $-0,14$ et $-0,06$ ) : les profits tirés par les élèves relativement faibles par rapport au niveau de leur classe sont sensiblement plus importants (un peu plus du double) que les pertes 
occasionnées par les élèves scolarisés dans une classe de niveau moyen inférieur au leur. Ce type de résultat invite à invoquer, au sein de la dimension «instructionnelle», des variables de type stimulation entre élèves, assez rarement étudiées dans la littérature.

Toujours pour avancer dans l'élucidation des mécanismes sous-jacents à l'influence des classes de niveau, il est maintenant intéressant de caractériser les classes par leur position relative au sein du collège : une classe «bonne» dans l'absolu (ou plus modestement dans l'échantillon!) peut en effet être la plus mauvaise classe d'un «bon» collège et réciproquement. D'où la construction d'une variable position de la classe, par calcul de l'écart entre son niveau moyen et celui des élèves du collège auquel elle appartient. Cette variable de position, continue (de moyenne 0,25 et d'écart-type 6,6), a ensuite été regroupée en catégories, trois modalités ayant été distinguées (classe située dans la moyenne du collège, classe très au-dessus - de plus d'un écart-type - ou très au dessous - à la même aune).

Les résultats du modèle 4 montrent que, toutes choses égales par ailleurs (et notamment à type de classe donné), le seul fait d'être scolarisé dans une classe située parmi les meilleures d'un collège accrô̂t significativement les progressions; certes, l'effet est minime $(+0,5$ point $)$, mais il est intéressant de souligner que cet effet vaut quel que soit le niveau moyen de la classe : même si celui-ci est faible, il suffit que la classe soit parmi les meilleures de l'établissement pour que l'effet négatif associé à la fréquentation d'une classe de niveau moyen faible soit en partie (il faut bien sûr comparer ce $+0,5$ point à des coefficients tels que $-2,8$ ou $-1,4$ ) contrebalancé par ce qui peut être interprété comme un effet d'étiquetage, mais qui se situe au niveau de l'établissement; on pourrait aussi invoquer un phénomène de nature différente tel que l'affectation des enseignants les plus efficaces aux classes considérées comme les meilleures.

\section{L'influence des cycles en 3 ans sur les progressions des élèves}

Parmi les modes de répartition des élèves, le fait de les affecter d'emblée dans un cycle aménagé, prévu pour durer 3 ans, est bien évidemment une solution drastique; certes, d'autres élèves mettront de fait 3 ans pour par-

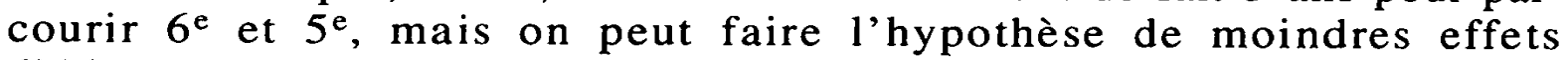
d'étiquetage négatifs. Le Tableau $v$ (modèle 5) donne les coefficients (9) attachés au type de $6^{\mathrm{e}}$ où l'élève a été affecté $\left(6^{\mathrm{e}}\right.$ standard dans un collège ne comportant pas de cycle aménagé, $6^{\mathrm{e}}$ standard dans un collège compor-

(9) Dans le Tableau $v$, seuls sont reportés les coefficients attachés aux variables contextuelles, ceux attachés aux caractéristi- ques individuelles de l'élève sont très proches de ceux présentés dans le Tableau III et ne sont pas repris ici. 
tant des classes de $6^{\mathrm{e}}-5^{\mathrm{e}}$ en 3 ans et enfin, $6^{\mathrm{e}}$ constituant la première année d'un cycle aménagé). On observe que des élèves initialement comparables connaissent des progressions quelque peu différenciées, l'écart principal opposant les élèves entrés dans une $6^{\mathrm{e}}$ standard (qu'elle soit située dans un collège organisant ou non par ailleurs un cycle aménagé) à ceux scolarisés dans une $6^{\mathrm{e}}$ aménagée : en fín de cycle, ces derniers obtiennent en moyenne environ 1 point de moins que les premiers.

TABLEAU V. - Effets des variables de scolarité sur les progressions au collège

\begin{tabular}{|l|c|c|}
\cline { 2 - 3 } \multicolumn{1}{c|}{} & Modèle 5 & Modèle 6 \\
\hline $6^{\mathrm{e}}$ standard - collège sans cycle aménagé/6 $6^{\mathrm{e}}$ aménagée & $+0,9(* * *)$ & \\
$6^{\mathrm{e}}$ standard - collège avec cycle aménagé/6 $/ 6^{\mathrm{e}}$ aménagée & $+1,4(* * *)$ & \\
& & $+2,9(* * *)$ \\
Cycle parcouru en 2 ans/cycle aménagé en 3 ans & $-2,4(* * *)$ \\
Cycle parcouru en 3 ans par redoublement $6 \%$ cycle aménagé en 3 ans & & $-0,9(*)$ \\
Cycle parcouru en 3 ans par redoublement $5 \%$ cycle aménagé en 3 ans & & \\
\hline
\end{tabular}

Le Tableau $v$ présente également (modèle 6) l'influence des différents itinéraires suivis par les élèves sur l'ensemble du cycle : cycle en 2 ans, cycle aménagé en 3 ans (pris comme référence), et cycle en 3 ans par redoublement de la $6^{\mathrm{e}}$ ou de la $5^{\mathrm{e}}$. À niveau initial (et autres caractéristiques personnelles) comparables, les élèves ayant réalisé leur cycle en deux ans ont le plus progressé, suivis, à près de 3 points, par les élèves scolarisés d'emblée en cycle aménagé; mais ce sont les élèves ayant redoublé la $5^{\mathrm{e}}(-0,9$ point par rapport aux élèves du cycle aménagé), et plus encore $\left(-2,4\right.$ points) ceux ayant redoublé la $6^{\mathrm{e}}$ qui réalisent les progressions les plus faibles. Ces résultats convergent avec les conclusions à présent bien établies quant aux effets négatifs du redoublement sur les progressions des élèves (Paul, 1996). La structure des coefficients montre que la scolarisation en cycle aménagé produit des effets moins négatifs que le redoublement. Alors que les élèves redoublants et ceux du cycle aménagé supportent vraisemblablement un étiquetage négatif comparable, les premiers refont le programme de l'année doublée alors que les seconds bénéficient d'un étalement dans le temps marquant a priori une meilleure continuité dans les apprentissages.

\section{La langue vivante comme vecteur de différenciation entre classes}

Enfin, au-delà des différenciations soit inscrites dans des dispositifs formels (cycle en 3 ans), soit existant de fait (classes de niveau et de degré d'hétérogénéité différents) sans que l'on en connaisse l'origine, il est apparu intéressant d'examiner dans quelle mesure la langue vivante choisie et le mode de groupement qui peut lui être associé est susceptible de constituer aussi un mode de différenciation des classes. On connaît en effet le 
rôle que peuvent jouer les options dans les stratégies de distinction des familles. Si, au niveau individuel, les élèves étudiant l'allemand obtiennent un score initial moyen de 111,4 (contre 100,3 pour les anglicistes et 99,2 pour les hispanisants), le niveau moyen des classes ne comptant que des germanistes est de 113,6 , loin devant les classes ne comptant que des anglicistes $(99,6)$, les classes «mixtes » étant de niveau intermédiaire.

Dans les modèles 7 à 11 , présentés dans le Tableau VI, on peut observer l'influence de la langue étudiée sur les progressions des élèves pendant les deux premières années du collège. Dans le modèle 7 , on observe les différences brutes en fin de cycle selon la langue étudiée, alors que les modèles 8 à 11 sont des modèles de progression intégrant les caractéristiques personnelles des élèves et (modèles 10 et 11) les caractéristiques des classes fréquentées.

TABlEAU VI. - Influence de la langue vivante sur les progressions des élèves en cours de cycle

\begin{tabular}{|c|c|c|c|c|c|}
\hline & Modèle 7 & Modèle 8 & Modèle 9 & Modèle 10 & Modèle 11 \\
\hline Constante & 99,6 & 27,4 & 76,8 & 68,0 & 76,8 \\
\hline $\begin{array}{l}\text { Score de début de } 6^{\mathrm{e}} \\
\text { Étranger/Français } \\
\text { Âge à l'entrée en } 6^{\mathrm{e}} \\
\text { Père «favorisé »/ } \\
\text { «défavorisé » } \\
\text { Mère cadre/autre } \\
\text { Garçon/fille } \\
\text { Anglais/espagnol } \\
\text { Allemand/espagnol } \\
\text { Niveau classe en } 6^{\mathrm{e}} \\
\text { Hétérogénéité classe } \\
\text { en } 6^{\mathrm{e}} \\
\text { GC1/GC5 } \\
\text { GC2/GC5 } \\
\text { GC3/GC5 } \\
\text { GC4/GC5 }\end{array}$ & $\begin{array}{l}+\quad 2,0(* * *) \\
+11,8(* * *)\end{array}$ & $\begin{array}{rr}+1,2 & (* * *) \\
+3,2 & (* * *)\end{array}$ & 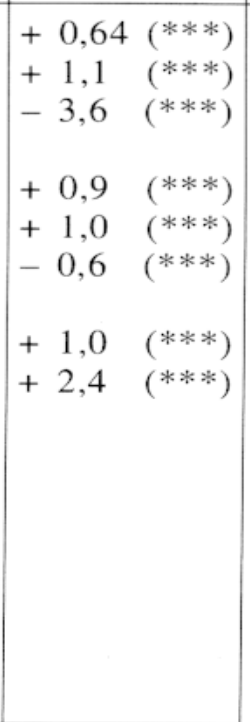 & 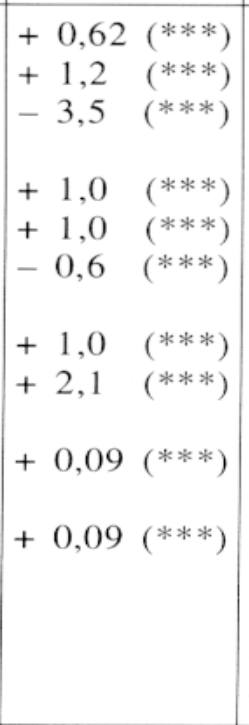 & $\begin{array}{ll}+0,62 & (* * *) \\
+1,2 & (* * *) \\
-3,5 & (* * *) \\
+1,0 & (* * *) \\
+1,0 & (* * *) \\
-0,6 & (* * *) \\
+1,0 & (* *) \\
+2,1 & (* * *) \\
& \\
& \\
& \\
-2,8 & (* * *) \\
-1,4 & (* * *) \\
-1,6 & (* * *) \\
-0,4 & (*)\end{array}$ \\
\hline $\begin{array}{l}\% \text { de variance } \\
\text { expliquée; } \mathrm{R}^{2}\end{array}$ & 4,5 & 50,3 & 52,5 & 52,8 & 52,8 \\
\hline
\end{tabular}

* GC1 classe faible à hétérogénéité faible

GC2 classe faible à hétérogénéité moyenne ou forte

GC3 classe moyenne à hétérogénéité faible

GC4 classe moyenne à hétérogénéité moyenne ou forte

GC5 classe forte à hétérogénéité faible, moyenne ou forte

Alors que de manière brute (modèle 7 ), les germanistes ont en moyenne les scores les plus élevés $(+12$ points par rapport aux hispanisants et +10 points par rapport aux anglicistes), les modèles 8 à 11 montrent que l'effet spécifique attaché à la langue est beaucoup plus modeste : le seul fait 
d'étudier l'allemand est associé à de meilleures progressions $(+2,4$ points), par rapport à des élèves de mêmes caractéristiques personnelles mais étudiant l'espagnol (les élèves étudiant l'anglais se situant en position intermédiaire, 1 point au-dessus des hispanisants et 1,4 point en dessous des germanistes).

Reste à savoir si cette plus forte progression est due au fait que cette langue étant choisie par de bons élèves, les germanistes se retrouvent regroupés dans des classes de bon niveau, ou bien aux vertus intellectuelles de l'apprentissage de la langue allemande ou encore à d'autres effets (étiquetage et attentes des enseignants, profil des enseignants affectés à ces classes, etc.). Les modèles 10 et 11 montrent que l'impact positif de l'allemand sur les progressions n'est que faiblement amoindri lorsque l'on prend en compte en outre le niveau moyen et l'hétérogénéité de la classe ; par ailleurs d'autres modèles ont pris en compte à la fois le fait d'étudier l'allemand et le type de classe dans lequel l'élève germaniste est scolarisé (en distinguant les classes de germanistes et les classes regroupant aussi des élèves apprenant une autre langue). Le Tableau VII donne les résultats obtenus (10):

\section{TABLEAU VII. - Effets de la langue étudiée et du type de classe sur les progressions des élèves}

\begin{tabular}{|l|c|c|}
\cline { 2 - 3 } \multicolumn{1}{c|}{} & Modèle 12 & Modèle 13 \\
\hline Anglais classe angliciste/espagnol & $+1,05(* * *)$ & $+1,20(* * *)$ \\
Anglais classe mixte germaniste/espagnol & $+1,65(* * *)$ & $+1,43(* * *)$ \\
Anglais classe mixte hispanisant/espagnol & $+0,85(* *)$ & $+0,94(* *)$ \\
Allemand classe germaniste/espagnol & $+3,43(* * *)$ & $+2,83(* * *)$ \\
Allemand classe mixte/espagnol & $+2,25(* * *)$ & $+2,01(* * *)$ \\
& & $+0,090(* * *)$ \\
Niveau moyen de la classe en 6 & & $+0,053(*)$ \\
\hline Hétérogénéité de la classe de 6 & & \\
\hline
\end{tabular}

On observe que des élèves de même niveau individuel initial et de mêmes caractéristiques personnelles étudiant l'allemand dans une classe de germanistes réalisent de loin les meilleures progressions; cet effet est pour une part lié à l'impact positif du niveau moyen de la classe comme le montre la comparaison avec le modèle 13 (le coefficient baisse de 3,43 à 2,83 points). La comparaison avec les élèves qui étudient l'allemand dans des classes mixtes montre qu'une part de cet effet $(0,82$ point par rapport à 2,83 points) est spécifique aux classes de germanistes (effets d'étiquetage positif de ces classes, caractéristiques plus favorables de leurs enseignants,...), et non à des phénomènes individuels (étiquetage positif

(10) Dans le Tableau VII, seuls sont reportés les coefficients attachés à la langue étudiée, ceux attachés aux autres caractéris- tiques individuelles de l'élève sont très proches de ceux présentés dans le Tableau vi et ne sont pas repris ici. 
des élèves germanistes, motivation particulière de leurs parents, effets cognitifs éventuels associés à l'apprentissage de la langue allemande,..). Il n'en reste pas moins que ces effets individuels existent comme le souligne le coefficient significatif $(+2,01$ points) attaché aux germanistes scolarisés dans des classes mixtes.

Sur la base de l'interprétation précédente, on pouvait faire l'hypothèse que les anglicistes scolarisés dans une classe comptant des germanistes bénéficieraient d'une part au moins des effets positifs associés aux classes comportant des germanistes; c'est effectivement le cas puisque le coefficient attaché à ces élèves (1,43 dans le modèle 13) est plus élevé que celui d'élèves comparables scolarisés avec des hispanisants $(+0,94)$. Le fait que les élèves anglicistes scolarisés avec des germanistes réalisent néanmoins des progressions légèrement inférieures à celles des élèves germanistes dans ces mêmes classes (coefficients respectifs de 1,43 et 2,01) conforte l'idée de phénomènes de nature individuelle, comme ceux évoqués précédemment au bénéfice des élèves apprenant l'allemand, même s'il reste un effet global positif à la fréquentation d'une classe où sont scolarisés des germanistes.

\section{Les classes de niveau et l'orientation des élèves en fin de cycle}

Si les élèves réalisent des acquisitions différentes selon le contexte de leur scolarisation, on peut s'attendre à ce que leur devenir à l'issue du cycle d'observation soit lui aussi différencié. Dans une première approche, on peut se positionner au moment de l'orientation et analyser le devenir des élèves à l'issue du cycle selon le niveau scolaire observé en fin de $5^{\mathrm{e}}$. Ceci permet d'évaluer d'éventuels effets d'étiquetage associés à la scolarisation dans telle ou telle classe : on ferait par exemple plus confiance, pour la suite des études, aux élèves des «bonnes»classes, et/ou ces élèves auraient une plus grande ambition ou une plus grande confiance dans leurs possibilités, pour la suite de leurs études. Dans une seconde approche, on peut se positionner cette fois à l'entrée en $6^{\mathrm{e}}$ et analyser quelles orientations obtiennent des élèves de caractéristiques (sociales, scolaires) initialement comparables; on explore ainsi de manière globale les effets de la scolarisation dans tel ou tel contexte, sans se poser la question de savoir si les différences de trajectoires éventuellement constatées relèvent d'inégalités dans les acquisitions réalisées ou de discriminations dans la procédure d'orientation elle-même. Examinons de manière synthétique ces deux perspectives.

La première s'appuie sur la modélisation de l'orientation obtenue en fin de cycle, sur la base des caractéristiques des élèves, de leur niveau scolaire et de leur âge en fin de cycle, considérées ici comme des variables de contrôle, ainsi que des variables décrivant le contexte de scolarisation 
qui sont ici la cible première de l'analyse. Pour la modélisation, nous avons utilisé une variable dichotomique opposant les élèves admis en $4^{\mathrm{e}}$ générale (quel que soit le temps mis pour y parvenir), soit $81,2 \%$ des élèves, aux autres situations, regroupant principalement les élèves admis en classe de $4^{\mathrm{e}}$ technologique.

Les modèles montrent tout d'abord la force des caractéristiques individuelles des élèves: en particulier, et sans surprise dans un système qui se veut méritocratique, le niveau scolaire individuel reste un paramètre essentiel. Cela dit, on observe aussi qu'à niveau scolaire comparable, les élèves âgés continuent de supporter un handicap considérable pour l'accès à une $4^{\text {e }}$ générale, de même que les enfants de milieux populaires, avec une continuité, depuis les enfants de chômeurs et d'ouvriers/agriculteurs qui ont les chances les plus faibles, jusqu'aux enfants dont le père est cadre supérieur. On remarque qu'à profession du père identique, les enfants dont la mère est cadre moyen ou supérieur bénéficient d'une orientation plus fréquente en $4^{\mathrm{e}}$ générale $(+6 \%$ environ). Les garçons continuent d'être orientés plus fréquemment que les filles $(+10 \%$ environ) vers une $4^{\mathrm{e}}$ technologique. Enfin, on remarque que les élèves de nationalité étrangère ne sont pas discriminés, à résultats scolaires et milieu social comparables, par rapport à leurs homologues français, puisqu'ils accèdent un peu plus souvent $\left(+8 \%\right.$ environ) à une $4^{\mathrm{e}}$ générale ; ces résultats concordent avec ceux obtenus par Vallet et Caillé (1996).

Les modèles prenant en compte les caractéristiques de la classe, sachant qu'il s'agit comme auparavant des caractéristiques de la classe de $6^{\mathrm{e}}$, montrent que le niveau moyen de la classe pas davantage que son hétérogénéité n'exercent d'effets notables sur l'orientation à valeur scolaire, âge et milieu social comparables. Cela dit, parler d' «âge comparable » a une signification limitée lorsque l'on compare des élèves ayant fréquenté une $6^{\mathrm{e}}$ standard et ceux ayant accédé à une $6^{\mathrm{e}}$ aménagée; en effet, le principe de cette dernière formule implique que les élèves qui la suivent prennent trois années au lieu de deux en général pour parcourir le cycle. Sauf à penser que tous ces élèves admis en cycle aménagé auraient redoublé une classe dans une organisation standard, on voit que le cycle aménagé génère un handicap spécifique dans la période d'orientation, sans toutefois que s'y surajoute un handicap supplémentaire dû à un éventuel effet d'étiquetage négatif. De même, aucun effet d'étiquetage (positif ou négatif) n'est observé sur la base de l'appartenance à une classe de niveau.

Envisageons maintenant l'orientation comme résultat du cycle, en reprenant l'analyse du devenir scolaire de l'élève (accès à une classe de $4^{\mathrm{e}}$ générale ou non), en nous situant cette fois à l'entrée en $6^{\mathrm{e}}$ et en explorant les différences de carrière qui pourraient résulter de son affectation dans un contexte donné (classe de niveau, type de collège); cette perspective est intéressante parce qu'elle est globale, même si elle ne permet pas en elle-même de distinguer ce qui se joue dans des mécanismes pédagogiques (progressions) et institutionnels (gestion de l'orientation). Les modèles esti- 


\section{Revue française de sociologie}

més font apparaître que d'une part la fréquentation d'une classe de niveau faible a un impact globalement négatif sur l'accès à une classe de $4^{e}$ générale, d'autre part que les chances d'accéder à cette classe sont à l'inverse d'autant plus élevées que l'élève fréquente une classe hétérogène.

Ces deux constats s'expliquent essentiellement par l'influence de ces deux variables sur les progressions des élèves, dans la mesure où les modèles précédents ont montré un effet de cette nature sur les progressions et leur absence d'influence au sein des processus d'orientation eux-mêmes. Il en découle que les classes homogènes faibles constituent un milieu de scolarisation défavorable quant à l'accès à une classe de $4^{\text {e }}$ générale. Un résultat comparable est observé pour l'accès à un cycle aménagé qui s'avère pénalisant sur le devenir scolaire des élèves essentiellement du fait de leurs moindres progressions.

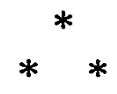

Dans ce texte, nous avons tenté d'évaluer les pratiques de classes de niveau, après avoir montré qu'elles étaient de fait assez répandues dans les collèges. D'une façon générale, des élèves de caractéristiques initiales comparables progressent d'autant mieux que le niveau moyen de leur classe est élevé ; ceci implique que ces élèves vont apprendre davantage lorsqu'ils appartiennent à une bonne classe et moins dans la situation inverse. Cette organisation pédagogique conduit donc à un accroissement des écarts de connaissances entre élèves par rapport à une situation de référence dans laquelle les élèves d'un même collège seraient répartis de manière aléatoire entre les différentes classes de l'établissement. Ce résultat contredit l'opinion courante selon laquelle les élèves faibles groupés dans une même classe profiteraient d'un enseignement ciblé sur leurs besoins. Alors que, au moins jusqu'au début des années 80 , la sociologie de l'école se focalisait sur l'influence des caractéristiques individuelles des élèves dans un système considéré de façon distanciée, ces résultats soulignent l'impact du contexte de scolarisation et militent pour une prise en compte concrète de ce qui se passe au sein des classes (nature des interactions pédagogiques, gestion du temps et couverture des programmes, attente des maîtres et effets d'étiquetage, etc.).

Ce contexte local résulte lui-même pour partie de décisions d'acteurs. Le texte présenté souligne que le contexte, au moins pour ce qui concerne le groupement des élèves, relève dans une mesure substantielle de choix d'organisation et non de contraintes de fonctionnement (la variété du recours à la pratique des classes de niveau en atteste). Ces choix d'organisation sont effectués au niveau local, et la pratique de classes de niveau ne suscite en général pas de conflit majeur dans la mesure où tant les convictions pédagogiques dominantes (l'hétérogénéité est nuisible) que les 
commodités en matière de groupement d'élèves pour les directions d'établissement militent en ce sens. Les parents d'élèves peuvent également appuyer ce type de pratiques, soit parce qu'ils pressentent, non sans raison, que leur enfant progressera mieux dans une bonne classe, soit parce qu'ils se laissent convaincre que leur enfant, de niveau un peu faible, gagnera à recevoir un traitement spécifique bien adapté à ses besoins dans une classe homogène.

Enfin, le constat selon lequel la position de l'élève par rapport au niveau moyen de sa classe exerce une influence sur ses progressions débouche sur un ensemble de questions de nature autant politique que sociologique. Dans la mesure où les enfants progressent d'autant mieux qu'ils sont scolarisés dans une bonne classe, on comprend que les familles aient tout intérêt à voir leur enfant scolarisé dans une classe de ce type. Cela dit, il est clair que la notion de «bonne classe étant relative, tous les élèves ne peuvent par définition avoir accès à ce type de classe. En outre, comme il s'avère aussi que les élèves de niveau inférieur au niveau de leur classe «gagnent» beaucoup plus que ne «perdent» les élèves situés au-dessus du niveau moyen de leurs condisciplines, le politique en charge de l'intérêt général devrait être conduit à promouvoir des classes hétérogènes. Il entre donc nécessairement en conflit avec l'intérêt individuel de chacune des familles qui tend à utiliser l'institution scolaire en fonction d'une logique de distinction.

Marie DURU-BELLAT

IREDU.CNRS - Université de Bourgogne 9, avenue Alain Savary, 21011 Dijon Cedex

Alain MINGAT

IREDU-CNRS - Université de Bourgogne 9, avenue Alain Savary, 21011 Dijon Cedex

\section{RÉFÉRENCES BIBLIOGRAPHIQUES}

Baudelot C., Establet R., 1971. - L'école capitaliste en France, Paris, Maspero.

Berends M., 1995. - «Educational stratification and students'social bonding to school », British journal of sociology of education, 16, 3, pp. 327-351.

Bourdieu P., Passeron J.-C., 1970. - La reproduction, Paris, Éditions de Minuit.

Bressoux P., 1994. - «Les recherches sur les effets écoles et les effets maîtres», Revue française de pédagogie, $\mathrm{n}^{\circ}$ 108, pp. 91-137.

Bressoux P., Coustère P., Leroy-Audouin C., 1997. - «Les modèles multiniveau dans l'analyse écologique : le cas de la recherche en éducation », Revue française de sociologie, 38,1 , pp. 67-95.

Bryk A., Raudenbush S., 1992. - Hierarchical linear models; applications and data analysis methods, London, Sage. 


\section{Revue française de sociologie}

Brophy J. E., Good T. L., 1974. - Teacher-students relationships : causes and consequences, New York, Holt Rinehart and Winston.

- 1986. - «Teacher behavior and student achievement», dans C. Wittrock Merlin (ed.), Handbook of research on teaching, New York, Macmillan, pp. 328-375 [3 $3^{\text {ème }}$ éd.].

Cherkaoui M., 1979. - Les paradoxes de la réussite scolaire, Paris, Presses Universitaires de France.

Cousin O., 1993. - «L'effet établissement; construction d'une problématique », Revue française de sociologie, 34, 3, pp. 395-419.

Dauber S., Alexander K., Entwisle D., 1996. - «Tracking and transitions through the middle grades : channeling educational trajectories », Sociology of education, vol. 69, pp. 290-307.

Davidson R., Mackinnon G., 1993. - Estimation and inference in econometrics, New York, Oxford University Press.

Duru-Bellat M., Mingat A., 1988. - «Le déroulement de la scolarité au collège : le contexte fait des différences », Revue française de sociologie, 29, 4, pp. 649-666.

- 1997. - La constitution de classes de niveau par les collèges et ses incidences sur les progressions et les carrières des élèves, Rapport pour la FEN, Cahier de 1'IREDU, $\mathrm{n}^{\circ} 59$, IREDU-CNRS.

Eder D., Felmee D., 1983. - «Contextual effects in a classroom : the impact of ability groups on student attention », Sociology of education, vol. 56, pp. 77-87.

Gamoran A., Berends M., 1987. - « The effects of stratification in secondary schools : synthesis of survey and ethnographic research , Review of educational research, 57, 4, pp. 415-435.

Gamoran A., Mare R. D., 1989s. - «Secondary school tracking and educational inequality : compensation, reinforcement, or neutrality?», American journal of sociology, 94, 5, pp. 1146-1183.

Good T. L., Marshall S., 1984. - «Do students learn more in heterogeneous or homogeneous groups? », dans P. Peterson, L. C. Wilkinson, M. Hallinan (eds.), The social context of instruction : group organisation and group processes, Orlando, Academic Press, pp. 1538.

Grisay A., 1990. - «Des indicateurs d'efficacité pour les établissements», Éducation et formations, $\mathrm{n}^{\circ} 22$, pp. 31-46.

- 1993. - «Le fonctionnement des collèges et ses effets sur les élèves de sixième et de cinquième ", Les dossiers éducation et formations, $\mathrm{n}^{\circ} 32$.

- 1993. - «Hétérogénéité des classes et équité éducative», Enjeux, no 30, pp. 69-95.

- 1994. - «Le fonctionnement des collèges et ses effets sur les élèves de sixième et de cinquième", Note d'information, pp. 94-17, Paris, DEP, ministère de l'Éducation nationale.

Keddie N., 1971. - «Classroom knowledge», dans F. D. Young (ed.), Knowledge and control, London, Collier Macmillan, pp. 133-160 [7 $7^{\grave{e ̀ m e}}$ éd.].

Kerckhoff A. C., 1986. - «Effects of ability grouping in British secondary schools», American sociological review, 51, 6, pp. 842-858.

Kulik C., Kulik J., 1982. - «Effects of ability grouping on secondary school students : a meta-analysis of evaluation findings ", Review of educational research, vol. 19, pp. 415428.

Metz M. H., 1978. - Classrooms and corridors : the crisis of authority in desegregated secondary schools, Berkeley, University of California Press.

Mingat A., 1984. - «Les acquisitions scolaires de l'élève au CP: les origines des différences? », Revue française de pédagogie, $\mathrm{n}^{\circ} 69$, pp. 49-64.

- 1987. - "Sur la dynamique des acquisitions à l'école élémentaire», Revue française de pédagogie, $\mathrm{n}^{\circ} 79$, pp. 5-14.

- 1991. - «Les activités de rééducation GAPP à l'école primaire. Analyse du fonctionnement et évaluation des effets», Revue française de sociologie, 32, 4, pp. 515-549. 
Oakes J., 1982. - «The reproduction of inequity : the content of secondary school tracking», Urban review, $\mathrm{n}^{\circ} 14$, pp. 107-120.

Pallas A. M., Entwisle D. R., Alexander K. L., Stluka M. F., 1994. - «Ability-group effects : instructional, social or institutional ? », Sociology of education, vol. 67, pp. 27-46.

Paul J.-J., 1996. - Le redoublement; pour ou contre ?, Paris, ESF.

Pelnard-Considère J., 1985. - «L'hétérogénéité des classes est-elle un handicap?», L'Orientation scolaire et professionnelle, 14, 1, pp. 3-21.

Rosenthal R., Jacobson L., 1972. - Pygmalion in the classroom, New York, Rinehart and Wilson.

Slavin R., 1987a. - "Ability grouping and student achievement in elementary schools : a best-evidence synthesis », Review of educational research, 57, 3, pp. 293-336.

- 1987b. - « Ability grouping in elementary schools : do we really know nothing until we know everything?», Review of educational research, 57, 3, pp. 347-350.

- 1990. - «Achievement effects of ability grouping in secondary schools : a best-evidence synthesis », Review of educational research, 60, 3, pp. 471-499.

Vallet L.-A., Caillé J.-P., 1996. - «Les élèves étrangers ou issus de l'immigration dans l'école et le collège français », Les dossiers d'éducation et formations, $\mathrm{n}^{\circ} 67$.

Welner K. G., Oakes J., 1996. - «Ability grouping : the new susceptibility of school tracking systems to legal challenges", Harvard educational review, 66, 3, pp. 451-470. 